\title{
Effect of Rhizobium Inoculation and Different Leaf Cutting Management on Seed Quality of Fenugreek (Trigonella foenum graecum L.)
}

\author{
Sumit Deswal*, S.K. Tehlan and V.S. Mor \\ Department of Vegetable Science, CCS Haryana Agricultural University, \\ Hisar-125004, Haryana, India \\ *Corresponding author
}

\begin{tabular}{|c|c|}
\hline & A B S T R A $\mathbf{T}$ \\
\hline & \multirow{6}{*}{$\begin{array}{l}\text { An experiment was conducted during Rabi season } 2012-2013 \text { for the evaluation different } \\
\text { levels of leaf cuttings and its impact on the seed quality fenugreek genotypes. Ten } \\
\text { genotypes were sown (November 12, 2012) with two sets of treatments (Rhizobium } \\
\text { inoculation and without Rhizobium) and three level of leaf cuttings (no cutting, one cutting } \\
\text { at } 30 \text { days, two cuttings at } 30 \text { and } 40 \text { days after sowing) and after attaining full maturity } \\
\text { seeds were harvested respectively and after that different seed quality parameters were } \\
\text { conducted to assess the effect of Rhizobium inoculation and three levels of leaf cutting on } \\
\text { seed quality of fenugreek genotypes. Maximum field emergence percentage was observed } \\
\text { for the genotype HM-348. The highest seedling length and dry weight was observed with } \\
\text { Rhizobium treatment in the genotype HM- } 291 \text { with no cutting. The maximum seedling } \\
\text { vigour index was observed with Rhizobium treatment by the genotype HM-291 with no } \\
\text { cutting. Minimum electrical conductivity }(0.069 \mu \text { S/cm/seed) was observed for genotype } \\
\text { Hisar Sonali without Rhizobium treatment with no cutting and HM-348 recorded } \\
\text { maximum seed density with Rhizobium treatment with no cutting. The genotype HM-348 } \\
\text { and HM-291 were observed superior in most of the seed quality parameters. Seed } \\
\text { inoculation with Rhizobium culture influenced the seed quality parameters in comparison } \\
\text { to no inoculation. }\end{array}$} \\
\hline Keywords & \\
\hline $\begin{array}{l}\text { Leaf cutting, } \\
\text { Rhizobium, } \\
\text { Seed quality. }\end{array}$ & \\
\hline Article Info & \\
\hline $\begin{array}{l}\text { Accepted: } \\
\text { 17 June } 2017 \\
\text { Available Online: } \\
\text { 10 July } 2017\end{array}$ & \\
\hline & \\
\hline
\end{tabular}

\section{Introduction}

Fenugreek (Trigonella foenum-graecum L.) is an annual plant in the family Fabaceae. It is also an important seed spice crop grown in Northern India during the Rabi season (winter season) for its leaves and seeds. It has various medicinal values and used to prevent some diseases including diabetes and knee pain. Fenugreek seeds (per $100 \mathrm{~g}$ ) are rich sources of protein ( $46 \%$ of DV), dietary fiber ( $98 \%$ DV),B vitamins, iron (186\% DV) and several other dietary minerals(USDA National Nutrient Database).Being a legume crop, it responds to inoculation with Rhizobium culture and supplemented with appropriate levels of nitrogen. Of all the nitrogen fixing microorganisms, Rhizobium has the maximum ability to fix nitrogen in association with legume crops and remainder benefits the succeeding crop, thereby improves crop and its soil productivity. Over recent years fenugreek is used as a dual purpose crop for greens leaf cutting as well for dry produce and there after living the crop for seed production has gained importance on account of the expectation of farmers to harness better returns. Seed viability and vigour are the most 
important attributes of seed quality. The quality seed is pre-requisite to enhance the production and productivity. Keeping in view of the above facts, the research was conducted to judge the effects of Rhizobium and leaf cutting levels on seed quality parameters of fenugreek genotypes.

\section{Materials and Methods}

Seed material comprised of ten genotypes fenugreek viz. HM-257, HM-273, HM-291, HM-293, HM-346, HM-348, HM-355, HM444, Hisar Suvarana and Hisar Sonali procured from the Department of Vegetable Science, Chaudhary Charan Singh Haryana Agricultural University, Hisar. The field experimental site was located at between $29.15^{\circ} \mathrm{N}$ latitude $75.69^{\circ} \mathrm{E}$ longitude with a mean altitude of $215 \mathrm{~m}$ above msl. Two sets of genotypes were under taken; one set was without Rhizobium inoculation while the second set inoculated with Rhizobium culture with three level of leaf cuttings: no cutting, one cutting at 30 days after sowing, two cutting at 30 and 40 day after sowing under both the sets of treatments, after the full maturity (May 2013) the seeds were collected respectively from each leaf cutting levels and the completely randomized design (CRD) was followed to conducted laboratory testing for the seed quality parameters. All recommended agronomic practices were followed timely for successful raising the seed crop and from each plot seeds were collected for measuring seed quality attributes. The observations were recorded on seed quality parameters were field emergence percentage, seedling length, dry weight, seedling vigour index, electrical conductivity, seed density as per the standard procedures.

\section{Results and Discussion}

With Rhizobium treatment, maximum field emergence percentage (Table 1) with no cutting was recorded for HM-348 (91.66 \%), at one cutting HM-346 (91.66 \%) and with two cuttings HM-346 (90.66\%). Without Rhizobium treatment the maximum field emergence percentage recorded for HM-291 (90.00\%) with no cutting, at one cutting HM$348(91.00 \%)$ and with two cuttings HM-348 $(92.00 \%)$ respectively. Similar observation were also recorded in Bengal gram, Moth Bean, Green gram, Peas by Pawar et al., (2014) and mentioned that seeds dressed with Rhizobium showed high seed germination and emergence percentage over control. Seedling length decreased significantly at one cutting and two cuttings as compared to no cutting in both the treatment. With Rhizobium treatment maximum seedling length (Table 2) was recorded by genotype HM-291(25.99 cm) with no cutting, HM-348 $(25.00 \mathrm{~cm})$ at one cutting and HM-257 $(23.90 \mathrm{~cm})$ at two cuttings respectively. Without Rhizobium treatment, maximum seedling length was recorded by genotype HM-348 $(24.11 \mathrm{~cm})$ with no cutting, Hisar Suvarna $(23.00 \mathrm{~cm})$ at one cutting and HM-291 $(21.90 \mathrm{~cm})$ with two cuttings respectively.

Badar et al., (2016) confirmed the similar finding in fenugreek that application of Rhizobium promotes the seedling length and other growth parameters. Similar results were also reported by Anitha et al., (2015) in fenugreek and suggested that increase in seedling length due to bolder seeds, having higher test weight.

Soundari et al., (2015) also recorded increase in root and shoot length in fenugreek that were treated with Rhizobium culture and proved to be best in enhancing root length. Dry weight decreased significantly in one cutting and two cuttings as compared to no cutting in both the treatment. With Rhizobium treatment maximum dry weight of seedling (Table 3) was recorded by genotype HM-291 (80.11 mg) with no cutting, while HM-348 
(79.11 mg) with one cutting and also in two cuttings $(80.02 \mathrm{mg})$ respectively. Without Rhizobium treatment maximum dry weight recorded for genotype HM-348 as $80.00 \mathrm{mg}$, $79.00 \mathrm{mg}$ and $78.67 \mathrm{mg}$ with no cutting, one cutting and two cuttings, respectively. These results were confirmed by Parakhia et al., (2000) in fenugreek that co-inoculation of Azotobacter with Rhizobium resulted in higher fresh and dry weight in comparison to control treatment. Sharangi et al., (2005) also reported that with Rhizobium inoculation highest dry matter was found compared to without inoculation. Similar results were also reported by Badar et al., (2016) and Anitha et al., (2015) in fenugreek. Soundari et al., (2015) found that Rhizobium inoculated plants showed increased dry weight in fenugreek. Kumar (2011) also reported similar effect of Rhizobium inoculation in pea. Seedling vigour index appeared slightly lower in two cuttings compared to no cutting and one cutting in both Rhizobium treatments. With Rhizobium treatment maximum seedling vigour index (Table 4) for genotype is HM-291 (7780.28) with no cutting, HM-348 with one cutting (7627.10) and two cuttings (7672.31) respectively.

Table.1 Effect of Rhizobium treatments and leaf cuttings levels on field emergence percentage among different genotypes of fenugreek

\begin{tabular}{|c|c|c|c|c|c|c|c|c|c|c|}
\hline \multirow{2}{*}{ Sr. no } & \multirow{2}{*}{ Genotype } & \multicolumn{4}{|c|}{ Without Rhizobium } & \multicolumn{4}{|c|}{ With Rhizobium } & \multirow[b]{2}{*}{$\begin{array}{c}\text { Overall } \\
\text { Mean }\end{array}$} \\
\hline & & $\begin{array}{c}\text { No } \\
\text { Cutting }\end{array}$ & $\begin{array}{c}\text { One } \\
\text { Cutting }\end{array}$ & $\begin{array}{c}\text { Two } \\
\text { Cutting }\end{array}$ & $\begin{array}{c}\text { Genotype } \\
\text { Mean }\end{array}$ & $\begin{array}{c}\text { No } \\
\text { Cutting }\end{array}$ & $\begin{array}{c}\text { One } \\
\text { Cutting }\end{array}$ & $\begin{array}{c}\text { Two } \\
\text { Cutting }\end{array}$ & $\begin{array}{c}\text { Genotype } \\
\text { Mean }\end{array}$ & \\
\hline 1 & HM-257 & 85.00 & 84.66 & 84.33 & 84.66 & 84.33 & 86.33 & 84.66 & 85.11 & 84.88 \\
\hline 2 & HM-273 & 85.66 & 86.33 & 85.66 & 85.88 & 84.66 & 86.00 & 86.66 & 85.77 & 85.83 \\
\hline 3 & HM-291 & 90.00 & 89.66 & 91.33 & 90.33 & 86.00 & 90.33 & 90.00 & 88.77 & 89.55 \\
\hline 4 & HM-293 & 86.66 & 87.00 & 86.66 & 86.77 & 89.00 & 86.33 & 87.00 & 87.44 & 87.11 \\
\hline 5 & HM-346 & 86.66 & 90.33 & 90.00 & 89.00 & 86.33 & 91.66 & 90.66 & 89.55 & 89.27 \\
\hline 6 & HM-348 & 89.00 & 91.00 & 92.00 & 90.66 & 91.66 & 91.33 & 90.00 & 91.00 & 90.83 \\
\hline 7 & HM-355 & 89.33 & 84.66 & 85.00 & 86.33 & 83.66 & 83.33 & 83.00 & 83.33 & 84.83 \\
\hline 8 & HM-444 & 83.66 & 89.33 & 88.66 & 87.22 & 90.66 & 90.33 & 90.00 & 90.33 & 88.77 \\
\hline 9 & Hisar Suvarna & 85.00 & 84.66 & 85.66 & 85.11 & 86.00 & 85.66 & 85.00 & 85.55 & 85.33 \\
\hline 10 & Hisar Sonali & 86.00 & 86.00 & 87.00 & 86.33 & 89.66 & 85.33 & 85.33 & 86.77 & 86.55 \\
\hline \multicolumn{2}{|c|}{ Cutting mean } & 86.70 & 87.36 & 87.63 & 87.23 & 87.20 & 87.66 & 87.23 & 87.36 & 87.30 \\
\hline
\end{tabular}

C.D. at $5 \%$ comparing two mean values of:

Rhizobium Treatment $(\mathrm{A})=\mathrm{NS} \quad$ Genotypes $(\mathrm{B})=0.348 \quad$ Cutting $(\mathrm{C})=0.191$

A X B $=0.492 \quad$ A X C $=0.269 \quad$ B X C $=0.603 \quad$ A X B X C $=0.852$

Table.2 Effect of Rhizobium treatments and leaf cuttings levels on seedling length $(\mathrm{cm})$ among different genotypes of fenugreek

\begin{tabular}{|c|c|c|c|c|c|c|c|c|c|c|}
\hline \multirow[t]{2}{*}{ Sr. no } & \multirow[t]{2}{*}{ Genotype } & \multicolumn{4}{|c|}{ Without Rhizobium } & \multicolumn{4}{|c|}{ With Rhizobium } & \multirow[b]{2}{*}{$\begin{array}{c}\text { Overall } \\
\text { Mean }\end{array}$} \\
\hline & & $\begin{array}{c}\text { No } \\
\text { Cutting }\end{array}$ & $\begin{array}{c}\text { One } \\
\text { Cutting }\end{array}$ & $\begin{array}{c}\text { Two } \\
\text { Cutting }\end{array}$ & $\begin{array}{c}\text { Genotype } \\
\text { Mean }\end{array}$ & $\begin{array}{c}\text { No } \\
\text { Cutting }\end{array}$ & $\begin{array}{c}\text { One } \\
\text { Cutting }\end{array}$ & $\begin{array}{c}\text { Two } \\
\text { Cutting }\end{array}$ & $\begin{array}{c}\text { Genotype } \\
\text { Mean }\end{array}$ & \\
\hline 1 & HM-257 & 20.09 & 19.89 & 19.53 & 19.84 & 20.54 & 20.27 & 23.90 & 21.57 & 20.70 \\
\hline 3 & HМ-291 & 22.54 & 22.27 & 21.90 & 22.24 & 25.99 & 19.53 & 18.99 & 21.50 & 21.87 \\
\hline 4 & HM-293 & 18.60 & 18.00 & 18.60 & 18.40 & 16.99 & 17.00 & 15.02 & 16.33 & 17.37 \\
\hline 5 & HM-346 & 15.98 & 15.80 & 15.78 & 15.85 & 16.40 & 16.06 & 16.05 & 16.17 & 16.01 \\
\hline 7 & HM-355 & 19.66 & 20.86 & 20.61 & 20.38 & 19.91 & 20.91 & 20.91 & 20.57 & 20.47 \\
\hline 8 & HM-444 & 19.31 & 19.31 & 19.40 & 19.34 & 20.02 & 19.99 & 20.00 & 20.00 & 19.67 \\
\hline 9 & Hisar Suvarna & 20.58 & 23.00 & 21.31 & 21.63 & 20.10 & 21.01 & 21.00 & 20.70 & 21.16 \\
\hline 10 & Hisar Sonali & 21.28 & 22.21 & 19.99 & 21.16 & 22.00 & 22.51 & 22.12 & 22.21 & 21.68 \\
\hline \multicolumn{2}{|c|}{ Cutting mean } & 20.14 & 20.06 & 19.53 & 19.91 & 20.69 & 20.32 & 19.76 & 20.25 & 19.16 \\
\hline
\end{tabular}

C.D. at $5 \%$ comparing two mean values of:

Rhizobium Treatment $(\mathrm{A})=0.034 \quad$ Genotypes $(\mathrm{B})=0.075 \quad$ Cutting $(\mathrm{C})=0.041$

A X B $=0.106$ A X C $=0.058 \quad$ B X C $=0.13 \quad$ A X B X C $=0.184$ 
Table.3 Effect of Rhizobium treatments and leaf cuttings levels on dry weight (mg) among different genotypes of fenugreek

\begin{tabular}{|c|c|c|c|c|c|c|c|c|c|c|}
\hline \multirow[t]{2}{*}{ Sr. no } & \multirow[t]{2}{*}{ Genotype } & \multicolumn{4}{|c|}{ Without Rhizobium } & \multicolumn{4}{|c|}{ With Rhizobium } & \multirow[b]{2}{*}{$\begin{array}{c}\text { Overall } \\
\text { mean }\end{array}$} \\
\hline & & $\begin{array}{c}\text { No } \\
\text { Cutting }\end{array}$ & $\begin{array}{c}\text { One } \\
\text { Cutting }\end{array}$ & $\begin{array}{c}\text { Two } \\
\text { Cutting }\end{array}$ & $\begin{array}{c}\text { Genotype } \\
\text { Mean }\end{array}$ & $\begin{array}{c}\text { No } \\
\text { Cutting }\end{array}$ & $\begin{array}{c}\text { One } \\
\text { Cutting }\end{array}$ & $\begin{array}{c}\text { Two } \\
\text { Cutting }\end{array}$ & $\begin{array}{c}\text { Genotype } \\
\text { Mean }\end{array}$ & \\
\hline 1 & HM-257 & 75.00 & 75.00 & 74.00 & 74.66 & 76.06 & 75.09 & 75.09 & 75.41 & 75.04 \\
\hline 2 & HM-273 & 71.00 & 70.82 & 69.78 & 70.53 & 71.73 & 70.10 & 70.81 & 70.88 & 70.70 \\
\hline 3 & HM-291 & 79.00 & 77.17 & 77.09 & 77.75 & 80.11 & 78.11 & 78.10 & 78.77 & 78.26 \\
\hline 4 & HM-293 & 77.92 & 77.74 & 76.98 & 77.54 & 79.39 & 77.97 & 76.15 & 77.83 & 77.69 \\
\hline 5 & HM-346 & 65.13 & 65.99 & 64.99 & 65.37 & 65.98 & 66.02 & 65.02 & 65.67 & 65.52 \\
\hline 6 & HM-348 & 80.00 & 79.00 & 78.67 & 79.22 & 79.93 & 79.11 & 80.02 & 79.69 & 79.45 \\
\hline 7 & HM-355 & 67.33 & 67.23 & 67.10 & 67.22 & 68.15 & 68.11 & 68.81 & 68.35 & 67.79 \\
\hline 8 & HM-444 & 69.99 & 70.01 & 70.02 & 70.00 & 70.02 & 70.02 & 70.03 & 70.02 & 70.01 \\
\hline 9 & Hisar Suvarna & 61.31 & 61.00 & 61.00 & 61.10 & 62.11 & 62.10 & 62.09 & 62.10 & 61.60 \\
\hline 10 & Hisar Sonali & 67.74 & 66.60 & 66.49 & 66.94 & 70.00 & 69.99 & 69.99 & 69.99 & 68.47 \\
\hline \multicolumn{2}{|c|}{ Cutting mean } & 71.44 & 71.05 & 70.61 & 71.03 & 72.35 & 71.66 & 71.61 & 71.87 & 71.45 \\
\hline
\end{tabular}

C.D. at $5 \%$ comparing two mean values of:

Rhizobium Treatment $(\mathrm{A})=0.055 \quad$ Genotypes $(\mathrm{B})=0.122 \quad$ Cutting $(\mathrm{C})=0.067$

A X B $=0.173$ A X C $=0.095 \quad$ B X C $=0.212 \quad$ A X B X C $=0.29$

Table.4 Effect of Rhizobium treatments and leaf cuttings levels on Vigour index among different genotypes of fenugreek

\begin{tabular}{|c|c|c|c|c|c|c|c|c|c|c|}
\hline \multirow[t]{2}{*}{ Sr. no } & \multirow[t]{2}{*}{ Genotype } & \multicolumn{4}{|c|}{ Without Rhizobium } & \multicolumn{4}{|c|}{ With Rhizobium } & \multirow[b]{2}{*}{$\begin{array}{c}\text { Overall } \\
\text { mean }\end{array}$} \\
\hline & & $\begin{array}{c}\text { No } \\
\text { Cutting }\end{array}$ & $\begin{array}{c}\text { One } \\
\text { Cutting }\end{array}$ & $\begin{array}{c}\text { Two } \\
\text { Cutting }\end{array}$ & $\begin{array}{c}\text { Genotype } \\
\text { Mean }\end{array}$ & $\begin{array}{c}\text { No } \\
\text { Cutting }\end{array}$ & $\begin{array}{c}\text { One } \\
\text { Cutting }\end{array}$ & $\begin{array}{c}\text { Two } \\
\text { Cutting }\end{array}$ & $\begin{array}{c}\text { Genotype } \\
\text { Mean }\end{array}$ & \\
\hline 1 & HM-257 & $7,200.14$ & $7,374.50$ & $7,104.00$ & $7,226.21$ & $7,303.60$ & $7,285.13$ & $7,215.78$ & $7,268.17$ & $7,247.19$ \\
\hline 2 & HM-273 & $6,816.64$ & $6,769.54$ & $6,698.88$ & $6,761.69$ & $6,957.81$ & $6,856.43$ & $6,797.76$ & $6,870.67$ & $6,816.18$ \\
\hline 3 & HM-291 & $7,592.48$ & $7,485.23$ & $7,462.31$ & 7,513.34 & $7,780.28$ & $7,576.41$ & $7,497.60$ & $7,618.10$ & $7,565.72$ \\
\hline 4 & HM-293 & $7,465.37$ & $7,463.04$ & $7,374.68$ & $7,434.37$ & $7,628.36$ & $7,492.97$ & $7,379.43$ & $7,500.26$ & $7,467.31$ \\
\hline 5 & HM-346 & $6,252.80$ & $6,400.36$ & $6,245.85$ & $6,299.67$ & $6,340.89$ & $6,337.92$ & $6,306.94$ & $6,328.58$ & $6,314.13$ \\
\hline 6 & HM-348 & $7,678.40$ & $7,593.01$ & $7,481.89$ & $7,584.43$ & $7,699.49$ & $7,627.10$ & $7,672.31$ & $7,666.30$ & $7,625.37$ \\
\hline 7 & HM-355 & $6,524.27$ & $6,521.63$ & $6,441.02$ & $6,495.64$ & $6,633.62$ & $6,674.09$ & $6,605.76$ & $6,637.82$ & $6,566.73$ \\
\hline 8 & HM-444 & $6,707.69$ & $6,725.01$ & $6,713.74$ & $6,715.48$ & $6,721.92$ & $6,792.91$ & $6,791.97$ & $6,768.93$ & $6,742.21$ \\
\hline 9 & Hisar Suvarna & $6,504.39$ & $6,459.63$ & $6,382.47$ & $6,448.83$ & $6,776.23$ & $6,845.81$ & $6,773.40$ & $6,798.48$ & $6,623.66$ \\
\hline 10 & Hisar Sonali & $5,886.40$ & $5,916.59$ & $5,855.39$ & $5,886.13$ & $6,000.56$ & $6,024.02$ & $5,960.96$ & $5,995.18$ & $5,940.65$ \\
\hline \multicolumn{2}{|c|}{ Cutting mean } & $6,862.86$ & $6,870.85$ & $6,776.02$ & $6,836.58$ & $6,984.28$ & $6,951.28$ & $6,900.19$ & $6,945.25$ & $6,890.91$ \\
\hline
\end{tabular}

C.D. at $5 \%$ comparing two mean values of:

Rhizobium Treatment $(\mathrm{A})=11.11 \quad$ Genotypes $(\mathrm{B})=28.884 \quad$ Cutting $(\mathrm{C})=13.607$

$\begin{array}{llll}\text { A X B }=35.134 & \text { A X C }=19.244 & \text { B X C }=43.03 & \text { A X B X C }=60.854\end{array}$

Table.5 Effect of Rhizobium treatments and leaf cuttings levels on electrical conductivity $(\mu \mathrm{S} / \mathrm{cm} / \mathrm{seed})$ among different genotypes of fenugreek

\begin{tabular}{|c|c|c|c|c|c|c|c|c|c|c|}
\hline \multirow[t]{2}{*}{ Sr. no } & \multirow[t]{2}{*}{ Genotype } & \multicolumn{4}{|c|}{ Without Rhizobium } & \multicolumn{4}{|c|}{ With Rhizobium } & \multirow[b]{2}{*}{$\begin{array}{c}\text { Overall } \\
\text { mean }\end{array}$} \\
\hline & & $\begin{array}{c}\text { No } \\
\text { Cutting }\end{array}$ & $\begin{array}{c}\text { One } \\
\text { Cutting }\end{array}$ & $\begin{array}{c}\text { Two } \\
\text { Cutting }\end{array}$ & $\begin{array}{c}\text { Genotype } \\
\text { Mean }\end{array}$ & $\begin{array}{c}\text { No } \\
\text { Cutting }\end{array}$ & $\begin{array}{c}\text { One } \\
\text { Cutting }\end{array}$ & $\begin{array}{c}\text { Two } \\
\text { Cutting }\end{array}$ & $\begin{array}{c}\text { Genotype } \\
\text { Mean }\end{array}$ & \\
\hline 1 & HM-257 & 0.125 & 0.125 & 0.122 & 0.124 & 0.131 & 0.132 & 0.131 & 0.131 & 0.128 \\
\hline 2 & HM-273 & 0.125 & 0.124 & 0.125 & 0.124 & 0.124 & 0.125 & 0.124 & 0.124 & 0.124 \\
\hline 3 & HM-291 & 0.180 & 0.180 & 0.180 & 0.180 & 0.170 & 0.170 & 0.169 & 0.170 & 0.175 \\
\hline 4 & HM-293 & 0.142 & 0.142 & 0.142 & 0.142 & 0.144 & 0.144 & 0.145 & 0.144 & 0.143 \\
\hline 5 & HM-346 & 0.087 & 0.087 & 0.080 & 0.084 & 0.090 & 0.090 & 0.090 & 0.090 & $\mathbf{0 . 0 8 7}$ \\
\hline 6 & HM-348 & 0.133 & 0.132 & 0.131 & 0.132 & 0.143 & 0.142 & 0.142 & 0.142 & 0.137 \\
\hline 7 & HM-355 & 0.119 & 0.118 & 0.117 & 0.118 & 0.118 & 0.117 & 0.118 & 0.118 & 0.118 \\
\hline 8 & HM-444 & 0.143 & 0.142 & 0.143 & 0.143 & 0.151 & 0.154 & 0.153 & 0.153 & 0.148 \\
\hline 9 & Hisar Suvarna & 0.113 & 0.113 & 0.113 & 0.113 & 0.112 & 0.112 & 0.111 & 0.112 & 0.113 \\
\hline 10 & Hisar Sonali & 0.069 & 0.070 & 0.098 & 0.079 & 0.097 & 0.098 & 0.098 & 0.098 & 0.088 \\
\hline \multicolumn{2}{|c|}{ Cutting mean } & 0.124 & 0.123 & 0.125 & 0.124 & 0.128 & 0.128 & 0.128 & 0.1282 & 0.126 \\
\hline
\end{tabular}

C.D. at $5 \%$ comparing two mean values of:

Rhizobium Treatment $(\mathrm{A})=0.003 \quad$ Genotypes $(\mathrm{B})=0.006 \quad \operatorname{Cutting}(\mathrm{C})=\mathrm{NS}$
A X B $=0.09$
$\mathrm{A} X \mathrm{C}=\mathrm{NS}$
$\mathrm{B} \times \mathrm{C}=\mathrm{NS}$
A X B X C = NS 
Table.6 Effect of Rhizobium treatments and leaf cuttings levels on seed density (g/cc) among different genotypes of fenugreek

\begin{tabular}{|c|c|c|c|c|c|c|c|c|c|c|}
\hline \multirow[t]{2}{*}{ Sr.no } & \multirow[t]{2}{*}{ Genotype } & \multicolumn{4}{|c|}{ Without Rhizobium } & \multicolumn{4}{|c|}{ With Rhizobium } & \multirow[b]{2}{*}{$\begin{array}{c}\text { Overall } \\
\text { mean }\end{array}$} \\
\hline & & $\begin{array}{c}\text { No } \\
\text { Cutting }\end{array}$ & $\begin{array}{c}\text { One } \\
\text { Cutting }\end{array}$ & $\begin{array}{c}\text { Two } \\
\text { Cutting }\end{array}$ & $\begin{array}{c}\text { Genotype } \\
\text { Mean }\end{array}$ & $\begin{array}{c}\text { No } \\
\text { Cutting }\end{array}$ & $\begin{array}{c}\text { One } \\
\text { Cutting }\end{array}$ & $\begin{array}{c}\text { Two } \\
\text { Cutting }\end{array}$ & $\begin{array}{c}\text { Genotype } \\
\text { Mean }\end{array}$ & \\
\hline 1 & HM-257 & 1.123 & 1.040 & 1.109 & 1.090 & 1.162 & 1.217 & 1.093 & 1.160 & 1.124 \\
\hline 2 & HM-273 & 1.430 & 1.405 & 1.380 & 1.410 & 1.477 & 1.441 & 1.331 & 1.420 & 1.411 \\
\hline 3 & HM-291 & 1.123 & 1.122 & 1.111 & 1.120 & 1.231 & 1.130 & 1.220 & 1.190 & 1.156 \\
\hline 4 & HM-293 & 1.446 & 1.321 & 1.343 & 1.370 & 1.243 & 1.415 & 1.109 & 1.260 & 1.313 \\
\hline 5 & HM-346 & 1.323 & 1.141 & 1.083 & 1.180 & 1.376 & 1.162 & 1.108 & 1.220 & 1.199 \\
\hline 6 & HM-348 & 1.443 & 1.442 & 1.433 & 1.440 & 1.538 & 1.533 & 1.444 & 1.510 & 1.472 \\
\hline 7 & HM-355 & 1.328 & 1.313 & 1.290 & 1.310 & 1.333 & 1.332 & 1.320 & 1.330 & 1.319 \\
\hline 8 & HM-444 & 1.348 & 1.342 & 1.322 & 1.340 & 1.454 & 1.455 & 1.331 & 1.410 & 1.375 \\
\hline 9 & Hisar Suvarna & 1.237 & 1.226 & 1.192 & 1.220 & 1.251 & 1.236 & 1.175 & 1.220 & 1.220 \\
\hline 10 & Hisar Sonali & 1.160 & 1.121 & 1.083 & 1.120 & 1.244 & 1.471 & 1.123 & 1.280 & 1.200 \\
\hline \multicolumn{2}{|c|}{ Cutting mean } & 1.300 & 1.250 & 1.230 & 1.260 & 1.330 & 1.340 & 1.230 & 1.300 & 1.278 \\
\hline
\end{tabular}

C.D. at $5 \%$ comparing two mean values of:

Rhizobium Treatment $(\mathrm{A})=0.028$ Genotypes $(\mathrm{B})=0.063$

A X B $=0.09 \quad$ A X C $=0.049$

$\mathrm{B} X \mathrm{C}=\mathrm{NS}$

$\operatorname{Cutting}(\mathrm{C})=0.034$

A X B X C $=\mathrm{NS}$

Without Rhizobium treatment genotype HM348 registered maximum vigour index as 7678.40, 7593.01 and 7481.89 with no cutting, one cutting and two cuttings respectively. Kumar et al., (2011) also observed that inoculation Rhizobium + Ensifer meliltoi has maximum vigour and alone application Rhizobium has positive and significant effect on fenugreek. Observation of table 5 revealed that, seed inoculated with Rhizobium culture showed slightly higher electrical conductivity except the genotype HM-291 and Hisar Suvarna as compared to no seed inoculation with Rhizobium culture at all the cutting levels respectively. Maximum electrical conductivity $0.180(\mu \mathrm{S} / \mathrm{cm} / \mathrm{seed})$ recorded for genotype HM-291 without Rhizobium, at all cutting levels respectively, and the minimum electrical conductivity (0.069) was observed for genotype Hisar Sonali without Rhizobium treatment with no cutting. Saxena et al., (1987) reported a negative relationship between electrical conductivity and the levels of reducing sugars in lechate with percent germination and other vigour and viability of vegetable seeds. Seed density in fenugreek seeds of different genotypes appeared slightly lower in two cuttings compared to no cutting and one cutting in both treatments respectively. With Rhizobium treatment maximum seed density (Table 6) recorded was for HM-348 with no cutting (1.538), one cutting (1.533) and with two cuttings (1.444) Without Rhizobium treatment maximum seed density (1.446) was recorded for HM-293 with no cutting, with one cutting (1.442) and two cuttings (1.443) maximum seed density recorded by genotype HM-348.

It is concluded that seed inoculation with Rhizobium culture improved all seed quality parameters compared to no seed inoculation with Rhizobium culture at all the cutting levels. All the characters decreased significantly at one cutting and two cuttings as compared to no cutting in both the Rhizobium treatment. The present investigation results and its derived inference summarized as that the inoculation with Rhizobium is beneficial, economical and ecological safe and better. Genotype HM-348 and HM-291 were observed superior in terms of seed quality parameters. Therefore, seed treatment with Rhizobium should be followed for obtaining higher leaf and seed yield along with good quality seed.

\section{References}

Anitha. M, D. V. Swami. And Salomi Suneetha D.R.2015. Seed yield and quality of fenugreek (Trigonella 
foenum-graecum L) cv. Lam Methi-2 as influenced by integrated nutrient management. The Bioscan.10 (1):103106.

Badar. Rabia, Rashid. Ummarah, Siddiqa. Aeysha, Kaleem. Maria. Khurshid. Hina, Atlaf.Sayeda. Sadaf, Kahid Harmain., Uroosa, Zamir. Tahreen, Mushtaque Wajiha., Yseen. Nida 2016: Application of biofertlizers for improving the growth of fenugreek plants (Trigonella foenum-graecum $\mathrm{L}$ ). $J$. of Pharmacognosy and Phytochemistry.5 (2):288-290

Kumar, Harish, Dubey, R.C., and Maheshwari D.K.2011.Effect of plant growth rhizobia on seed germination, growth promotion and suppression of Fusarium wilt of fenugreek. Crop Protection.30, 1396-1403

Kumar, Jitender 2011.Effect of Phosphorous and Rhizobium inoculation on the growth, nodulation and yield of garden pea (Pisum Sativum) cv-Matar Agetha6. Legume Research.34 (1):20-25

Parakhia, A.M., Akbari, L.F. and Andharia, J.H 2000. Seed bacterization for better quality and more yield of fenugreek Gujarat Agri. Uni. Res. J.25 (2):34-38

Pawar, Vaishali. A., Pawar,Pooja.R., Bhosale,Ashok.M., Chavan., Sourabh V.2014. Effect of Rhizobium on seed Germination and growth of plants. $J$. of Academia and Industrial Research. 3(2):84-88.

Saxena, O.P., Singh, G., Pakeeraiah, T. and Panday, N.1987. Seed deterioration studies in some vegetable seeds. Acta Horticulturae, 215: 39-44.

Sharangi. A.B. Thapa. U., Pariari. A, Mandal. A.R., Chatterjee. $\mathrm{R}$ and Sivkumar. T.2005. Response of nitrogen, Rhizobium and cutting management of nodules behavior of fenugreek. Legume Res., 28 (3):184-188.

Soundari, M, Vaithiyanathan, T. and Sundaramoorthy, P.2015.Response of fenugreek (Trigonella foenum-graecum L.) to Rhizobium inoculation. Int. Letters of Natural Science.6:9-11

USDA National Nutrient Database May 2016. Fenugreek seed per 100g. Conde Nast Nutrition data.com.

\section{How to cite this article:}

Sumit Deswal, S.K. Tehlan and Mor, V.S. 2017. Effect of Rhizobium Inoculation and Different Leaf Cutting Management on Seed Quality of Fenugreek (Trigonella foenum graecum L.). Int.J.Curr.Microbiol.App.Sci. 6(7): 1045-1050. doi: https://doi.org/10.20546/ijcmas.2017.607.125 